\title{
Relevance of porosity and surface chemistry of superactivated carbons in capacitors
}

\author{
D. Lozano-Castelló ${ }^{\text {a), * }, ~ J . ~ P . ~ M a r c o-L o z a r ~}{ }^{\text {a) }}$, M. J. Bleda-Martínez ${ }^{\text {a) }}$, F. Montilla ${ }^{\text {b) }}$, E. Morallón ${ }^{\text {b) }}$, \\ A. Linares-Solano ${ }^{\text {a) }}$ and D. Cazorla-Amorós ${ }^{\text {a) }}$
}

\begin{abstract}
We show, through some examples, that chemical activation by alkaline hydroxides permits the preparation of activated carbons with tailored pore volume, pore size distribution, pore structure and surface chemistry, which are useful for their application as electrodes in supercapacitors. Examples are presented discussing the importance of each of these properties on the double layer capacitance, on the kinetics of the electric double-layer charge-discharge process and on the pseudo-capacitative contribution from the surface functional groups or the addition of a conducting polymer.
\end{abstract}

KEYWORDS : Activated carbon, Conducting polymer, Hybrid materials

\section{Introduction}

The performance of activated carbons (ACs) as electrical double layer capacitors (EDLC) is strongly dependent on a complex combination of properties which include carbon material properties, preparation method of the electrode and electrolyte characteristics (such as ions dimensions, dielectric constant, etc. $)^{1)}$. The proper selection of these properties will allow to optimize the EDLC.

The objective of this work is to present a brief summary of the preparation and characterization of porous carbon materials with different properties (apparent surface area, pore volume, pore size distribution, surface chemistry and porous structure) by chemical activation with alkaline hydroxides of different precursors and to show some examples about the relevance of these properties on the double layer capacitance, on the kinetics of the electric double-layer chargedischarge process, and on the pseudo-capacitative contribution from the surface functional groups or the addition of a conducting polymer.

\section{Chemically activated carbons: Porous texture and surface chemistry characterization}

A wide series of chemically activated carbons can be prepared using different carbonaceous precursors (i.e., anthracite, lignite, subbituminous coal, eucalyptus wood, coconut shell, beech wood and carbon fibre) with potassium and sodium hydroxides (using alkaline hydroxide/carbon ratios from $1 / 1$ up to $7 / 1$ ), followed by a heat treat- ment stage at $750{ }^{\circ} \mathrm{C}$ and finally by a washing step with hydrochloric acid and water.

As an example, Fig. 1 presents the $\mathrm{N}_{2}$ adsorption isotherms at $77 \mathrm{~K}$ corresponding to some of these carbon precursors used that were prepared with $\mathrm{NaOH}(3 / 1)$. A great variability is observed both on the adsorption capacities and on the shape of the isotherms, which emphasises the important role played by the precursor. The results obtained with $\mathrm{KOH}$ activation (not shown here) point out that the porosity development of a given precursor is different for $\mathrm{NaOH}$ and $\mathrm{KOH}$. It has been seen that, in general, the development of porosity for precursors with low structural order (i.e., original eucalyptus precur-

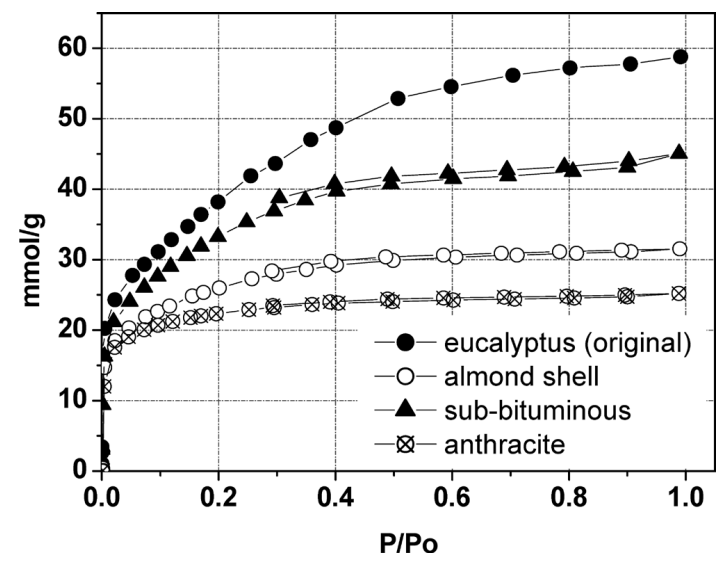

Fig. $1 \mathrm{~N}_{2}$ adsorption isotherms at $77 \mathrm{~K}$ corresponding to different precursors activated with $\mathrm{NaOH}(3 / 1)$ at $750^{\circ} \mathrm{C}$.

\footnotetext{
* Corresponding Author, E-mail: d.lozano@ua.es 


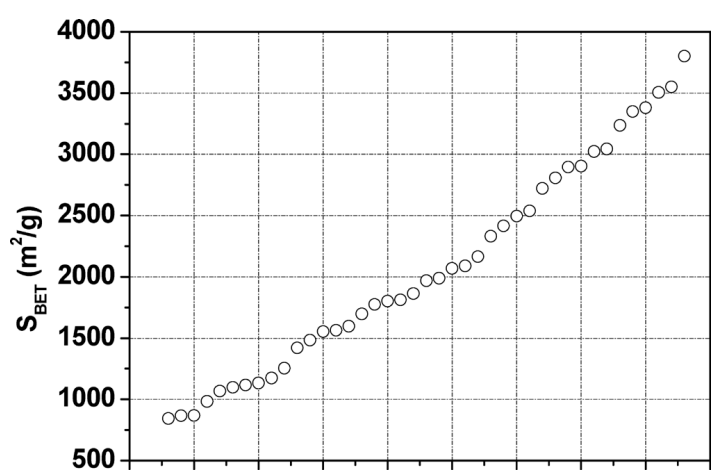

Fig. 2 Apparent BET surface area corresponding to selected AC prepared by alkaline hydroxide activation of several precursors under different experimental conditions.

sors (non-carbonized)) is better with $\mathrm{NaOH}$ than $\mathrm{KOH}$, whereas the opposite is observed for the more ordered ones (i.e., anthracite) $)^{2), 3)}$.

Fig. 2 shows the wide range of apparent BET surface area (from $700 \mathrm{~m}^{2} / \mathrm{g}$ up to around $3800 \mathrm{~m}^{2} / \mathrm{g}$ ) covered with the series of ACs prepared in the present work. It should be remarked that this method also allows us to prepare materials with different pore structure ${ }^{2)}$.

As it will be discussed in the next section, in addition to the apparent surface area and micropore volume, the pore size distribution (PSD) of the ACs is also a key parameter for their use as supercapacitors. Fig. 3 contains the pore size distribution obtained by density functional theory (DFT) for a selection of materials. In Fig. 3a) and b), it can be seen that for a given precursor (in that case beech wood), the higher the alkaline hydroxide/carbon ratio the wider the resulting PSD. Comparing Fig. 3b) and c), a low-ordered precursor (beech wood) and a much more ordered precursor (anthracite), it is observed that for the beech wood, the porosity distribution is wider for $\mathrm{NaOH}$ activation than for $\mathrm{KOH}$ activation, whereas for the anthracite, the porosity distribution is wider for the $\mathrm{KOH}$ activated carbons ${ }^{3)}$.

Another important property of the AC is the surface chemistry. Fig. 4 includes, as an example, the temperature programmed desorption (TPD) curves corresponding to $\mathrm{CO}$ and $\mathrm{CO}_{2}$ desorption in a carbonized eucalyptus wood, before and after activation with $\mathrm{KOH}$ and $\mathrm{NaOH}$. From these results and those obtained with other precursors (not shown here) it can be concluded that: i) chemical activation with alkaline hydroxide produces a significant increase on the oxygen content and; ii) $\mathrm{KOH}$ shows a higher surface oxygen groups development than $\mathrm{NaOH}$, specially for oxygen evolving as $\mathrm{CO}$.

Thus, by selecting the activation conditions, porous carbon materials with tailored pore volume, PSD, pore structure and surface chemistry can be prepared, to optimize their performance as EDLC.

\section{Double layer capacitance and porosity (pore volume and pore size distribution)}

It is known that double layer capacitance of carbon materials depends on the porosity, and some correlations with parameters such
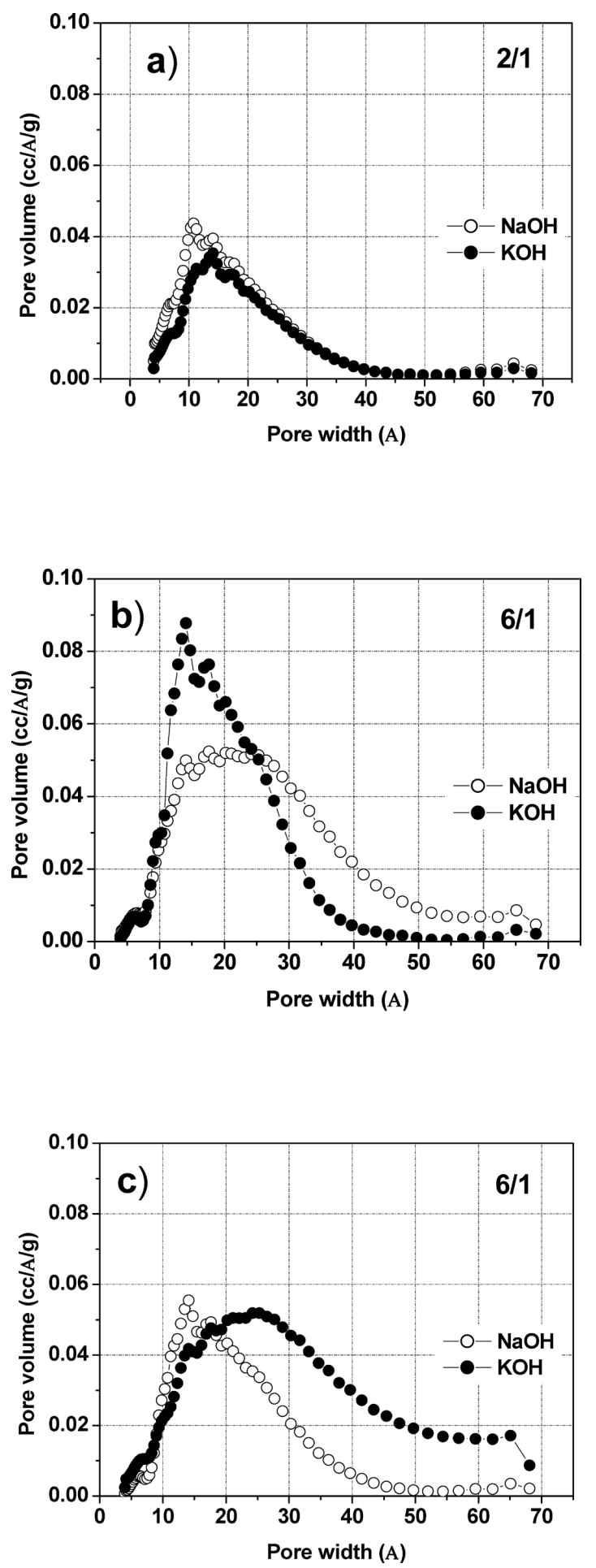

Fig. 3 DFT porosity distribution of ACs prepared by $\mathrm{NaOH}$ and $\mathrm{KOH}$ activation of (a) beech wood (ratio alkaline hydroxide/ carbon 2/1), (b) beech wood (ratio alkaline hydroxide/carbon 6/1) and (c) anthracite (ratio alkaline hydroxide/carbon 6/1).

as apparent surface area or the micropore volume have already been published ${ }^{4-7)}$. In general, the higher the surface area of carbon materials, the higher is the capacitance value. However, important deviations from this trend have been observed ${ }^{4)}$, confirming that there are other important factors that contribute to double layer capacitance. To show this general trend, Fig. 5 shows the relationship between the 

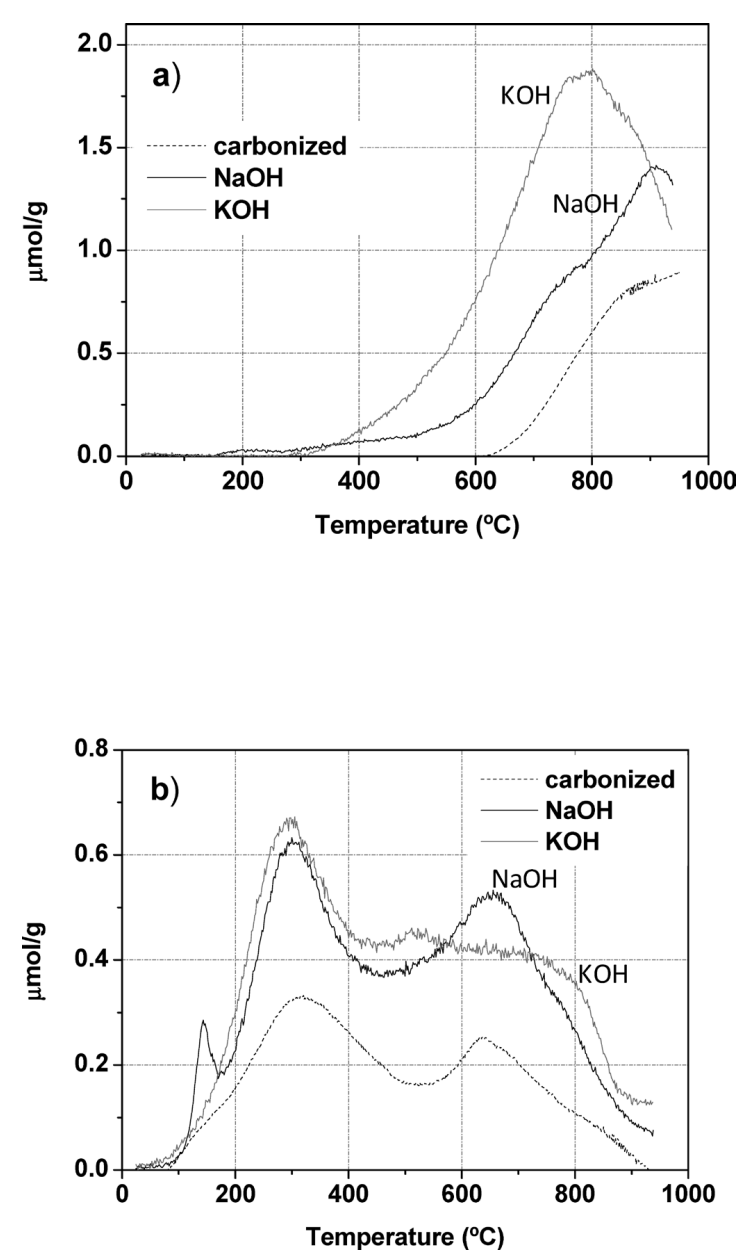

Fig. 4 TPD curves corresponding to a carbonized eucalyptus wood before and after activation with $\mathrm{KOH}$ and $\mathrm{NaOH}$. a) $\mathrm{CO}$ desorption profile and b) $\mathrm{CO}_{2}$ desorption profile.

BET surface area and the specific capacitance, measured in $\mathrm{H}_{2} \mathrm{SO}_{4}$, for a number of activated carbons with different porosities, structure and surface chemistry. This series of materials have been prepared using different precursors, such as an anthracite (denoted as A), general purpose carbon fibres (denoted as D) and high performance carbon fibres (denoted as $\mathrm{H}$ ), which have been activated by chemical activation with $\mathrm{KOH}$ (denoted as $\mathrm{K}$ ) and $\mathrm{NaOH}$ (denoted as $\mathrm{Na}$ ). The preparation, characterization and capacitance measurements details are included elsewhere ${ }^{8)}$.

The capacitance values for the general purpose activated carbons fibres (ACFs) activated both with $\mathrm{NaOH}$ or $\mathrm{KOH}$ follow a linear relationship with BET surface area, except for the samples with the lowest surface areas (i.e., those with BET surface areas below $500 \mathrm{~m}^{2} / \mathrm{g}$ ). Such exception related with an ion sieving effect due to the small pore size of these materials ${ }^{4), 9)}$, shows the need of adjusting the pore size of the carbon material to the size of the electrolyte ${ }^{10)}$. Contrarily, in the case of the anthracite and Hexcel-derived ACs, the capacitance goes through a maximum, which is especially remarkable for the former.

The sample with the largest capacitance corresponds to the acti-

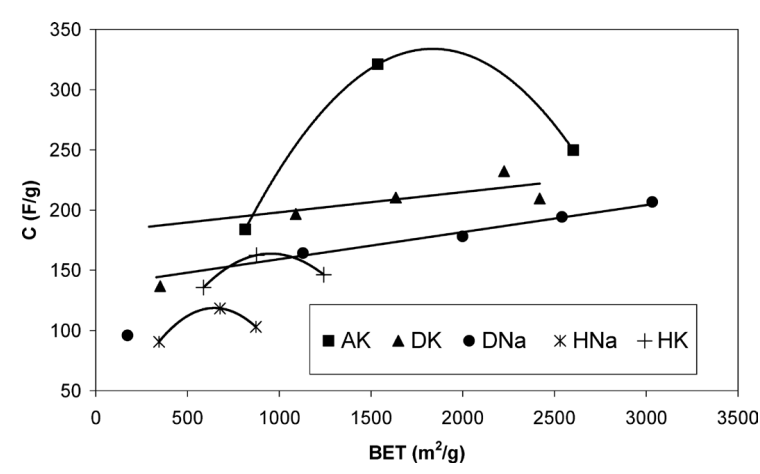

Fig. 5 Electric double capacitance versus apparent BET surface area for samples prepared by chemical activation with $\mathrm{KOH}$ and $\mathrm{NaOH}$ (denoted as $\mathrm{K}$ and $\mathrm{Na}$, respectively) of different precursors (anthracite, general purpose and high performance carbon fibres, denoted as $\mathrm{A}, \mathrm{D}$ and $\mathrm{H}$, respectively).

vated anthracite, reaching a value of $321 \mathrm{~F} / \mathrm{g}$. It should be emphasized that in this last sample, the porosity is sufficiently well developed to remove the ion-sieving effect mentioned before. It must be noted that the sample with the highest capacitance has a BET surface area of only about $1500 \mathrm{~m}^{2} / \mathrm{g}$. This means that although porosity is a key parameter in this process, there are other factors which influence in a considerable extent the behaviour of the ACs in EDLC. As it is described in the next section, apart from porosity, surface chemistry of the porous carbons is also a relevant parameter.

\section{Role of surface chemistry on electric double layer capacitance}

Surface oxygen groups on carbon materials decompose upon heating producing $\mathrm{CO}$ and $\mathrm{CO}_{2}$ at different temperatures ${ }^{11)}$. The amount of surface oxygen functional groups can be measured by TPD experiments. To study the effect of the surface chemistry on the capacitance values, a large number of porous carbons (powder and fibres) were prepared in our laboratory from different precursors and using several activating agents. The detailed characterization results have been discussed elsewhere ${ }^{8)}$. As a summary, it can be said that samples with a wide range of surface oxygen groups were available, reaching for some samples prepared by $\mathrm{KOH}$ activation a total oxygen content of $5000 \mu \mathrm{mol} / \mathrm{g}$.

To analyze the influence of the oxygen surface chemistry on the capacitance values, we calculated the capacitance per BET surface area and we plotted in Fig. 6 such parameter ratio with the oxygen surface group evolving as $\mathrm{CO}$, assessed from $\mathrm{TPD}^{8)}$. It is seen that the correlation is excellent for all the samples except for one prepared from high performance carbon fibres ${ }^{8)}$. This well defined correlation, deduced for a large number of samples prepared from different raw materials and using different preparation methods, confirms that the CO-type groups of the porous carbons have a positive contribution to the capacitance. This result was also proposed from studies done 
with a given porous carbon after a selective modification of the surface chemistry ${ }^{12), 13)}$. It should be remarked that such capacitance per BET surface area has not a good correlation neither with the number of oxygen groups desorbing as $\mathrm{CO}_{2}$ nor with the total oxygen content calculated as $2 \mathrm{CO}_{2}+\mathrm{CO}$. The $\mathrm{CO}$-type surface complexes are mainly hydroxyl, carbonyl or quinone groups which can undergo the wellknown mechanism for the quinone/hydroquinone redox pair:

$$
>\mathrm{C}_{x} \mathrm{O}+\mathrm{H}^{+}+\mathrm{e}^{-} \rightleftarrows \mathrm{C}_{x} \mathrm{OH}
$$

In the series of samples presented in Fig. 6, we used the ACs without any treatment after activation. Considering that the activation temperature is usually higher than $700^{\circ} \mathrm{C}$, some oxygen groups that desorb at lower temperatures, e.g., anhydrides, will not remain in the carbon material and hence, the effect of these oxygen groups in capacitance cannot be well studied. To analyze the effect of these oxygen groups, a deep preparation and characterization of samples with tailored surface chemistry were carried out in our laboratory, as explained elsewhere ${ }^{14)}$. Thus, two chemically ACs were oxidized with $\mathrm{HNO}_{3}$ and thermally treated in $\mathrm{N}_{2}$ flow at different temperatures in order to obtain porous carbon materials with different amounts of surface oxygen complexes. Additionally, a thermal treatment in $\mathrm{H}_{2}$ was also carried out, with a subsequent treatment in air flow at $450{ }^{\circ} \mathrm{C}$. Then, materials with very similar porous texture and widely different surface chemistry were compared. The surface chemistry of the resulting materials was systematically characterized by TPD and X-ray photoelectron spectroscopy (XPS) measurements. The results pointed out that some samples had an important content of carboxylic anhydride groups. It is known that the thermal decomposition of each carboxylic anhydride produces one $\mathrm{CO}$ molecule and one $\mathrm{CO}_{2}$ molecule at close temperatures. Thus, the amount of $\mathrm{CO}$ corresponding to anhydrides can be obtained from the deconvolution of TPD profiles.

In that study, galvanostatic and voltammetric techniques were used to deepen into the characterization of the surface oxygen complexes of that series of samples ${ }^{14)}$. A clear correlation was found between capacitance and the CO-type groups (capacitance vs. CO-type groups plot), but the sample without further thermal treatment or heat-treated below $450{ }^{\circ} \mathrm{C}$, exhibited an upwards deviation. The fact that those samples contained a significant amount of anhydrides, pointed out that this type of groups could be responsible for the enhancement in capacitance.

The main observed features from the steady cyclic voltammograms were presumably assigned to redox processes associated to CO-type groups (broad peak below 0.6 V RHE), which proceeds through a one electron transfer quinone/hydroquinone mechanism. Moreover, in the case of samples with high content of carboxylic anhydrides groups, a peak at about $0.63 \mathrm{~V}$ RHE was observed, and this peak disappeared almost completely for other samples, which was thermal treated at temperature higher than $450{ }^{\circ} \mathrm{C}$, where those surface

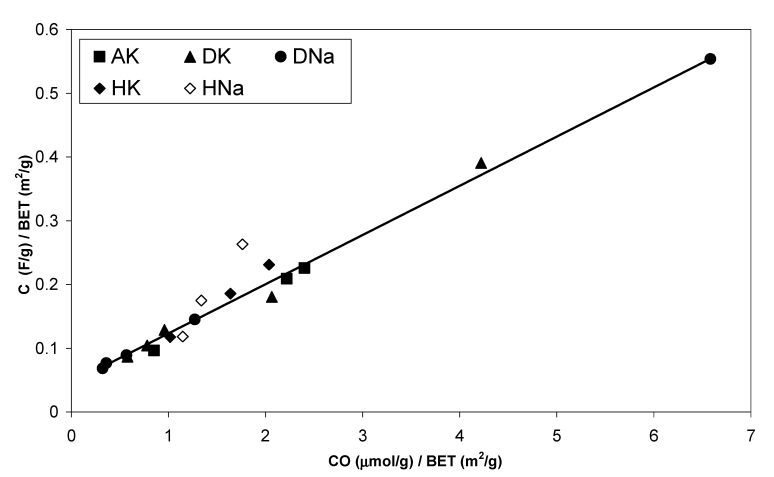

Fig. 6 Capacitance divided by BET surface area versus $\mathrm{CO}$ content divided by BET surface area for samples prepared by chemical activation with $\mathrm{KOH}$ and $\mathrm{NaOH}$ (denoted as $\mathrm{K}$ and $\mathrm{Na}$, respectively) of different precursors (anthracite, general purpose and high performance carbon fibres, denoted as A, D and $\mathrm{H}$, respectively).

oxygen groups were removed. With these results, it was suggested that anhydrides undergo a redox reaction at this potential. This reaction should be somehow different from that occurring in carbonyls or quinone groups, whose redox process occur at lower potentials. In that study ${ }^{14)}$, it was proposed a two electron mechanism for the redox process corresponding to anhydrides. According to that mechanism, each anhydride group would contribute with two electrons in the redox process. This aspect was considered in the capacitance vs. COtype groups plot. Each carboxylic anhydride contributed to capacitance with two electrons, so the amount of anhydrides was taken into account twice. The amount of CO desorbing groups in the corrected plot, corresponded to the CO desorbed in TPD experiments plus the part corresponding to anhydrides (obtained from the deconvolution). In this type of corrected plot, all the samples fitted much better to the linear trend.

From the above study ${ }^{14)}$ it has been shown, that the surface oxygen groups have, at least, a twofold relevant contribution to the total capacitance of porous carbons; i) as previously mentioned the surface oxygen groups have an important contribution to the capacitance through faradic processes which involve one or two electron transfer reactions and ii) the surface oxygen groups (or the dangling bonds created after decomposition, in inert atmosphere or in a $\mathrm{H}_{2}$ atmosphere), determine the wettability of aqueous electrolyte; thus, their presence on the carbon surface is essential to take profit of the large double layer contribution to the capacitance of the porous carbons which is associated to their high porosity. Further studies in organic medium are being done to understand the role of these functionalities in the capacitance.

\section{Influence of porous structure and pore size distri- bution on the kinetics of double layer formation}

As it has been described in Section 3, it is well-known that, in general, the larger the surface area that an EDLC can provide for 
adsorption of ions on electrodes, the more energy can be stored in the EDLC. However, detailed analyses using porous carbons with tailored porosity have demonstrated that not all of the pores are effective in the charge accumulation. For example, it has been previously mentioned that the very narrow micropores do not contribute to the total double-layer capacitance because of a molecular sieving effect ${ }^{4), 9)}$. Most of the studies have been performed using low currents or low scan rates. However, from an application point of view, it is very interesting to know how the materials behave at high charge/discharge rates, because the accessibility of the porosity will also depend upon the process rate. Thus, it is very important to study the kinetics of the double-layer formation using porous materials to guarantee that they can be used in high-power applications.

Our research group carried out a systematic study about the influence of the porous structure and pore size distribution on the kinetics of the electric double-layer charge-discharge process ${ }^{15)}$. This study was performed using electrochemical impedance spectroscopy. To cover a wide range of apparent surface area, pore size distribution, and pore structure, a series of ACs and ACFs were prepared from an anthracite and two different carbon fibres by different activation methods $\left(\mathrm{KOH}\right.$ and $\mathrm{CO}_{2}$ ). In addition, two commercial ACs from Maxsorb were also studied.

To quantify the level of reduction of capacitance, capacitance loss percentages (CLPs) from the starting value (at the lowest frequency, where there is enough time for the formation of the double layer in the porosity) to the value at around $0.02 \mathrm{~Hz}$ frequency were calculated for all of the samples. Fig. 7 plots the CLPs versus the percentage of narrow microporosity $\left(<0.7 \mathrm{~nm}\right.$ ) for some samples in $\mathrm{Na}_{2} \mathrm{SO}_{4}$ medium, as an example of the results obtained. The upper ordinate axis includes the average pore size, which permits to follow the discussion more easily. A clear linear relationship is observed; that is, the higher the percentage of narrow microporosity, the higher the CLP ${ }^{15)}$. That means that, from a kinetic point of view, porous carbon materials with a wide mean pore size have better performance; i.e., the porosity is more easily accessible for the electrolyte even at high chargedischarge rates. It must be remarked that quite different trends are obtained for the ACs and ACFs. In the case of the ACs, the widening of the porosity does not produce a considerable improvement of the kinetic behaviour. However, in the case of the ACF, the CLP decreases importantly when the porosity becomes wider. Thus, for $\mathrm{Na}_{2} \mathrm{SO}_{4}$ electrolyte, ACFs seem to be more useful in high-power applications than ACs. The fact that a widening of the porosity produces better kinetic behaviour in $\mathrm{ACF}$ than in $\mathrm{AC}$ in $\mathrm{Na}_{2} \mathrm{SO}_{4}$ medium is most likely due to the existence of a smaller tortuosity of networks of porosity in ACF than in AC, which makes the ACF have a shorter path length for the ions to move than in the case of $\mathrm{AC}^{15}$.

An analogous analysis has been performed in $\mathrm{H}_{2} \mathrm{SO}_{4}$ medium ${ }^{15}$. A widening of the porosity produces an improvement of the kinetic be-

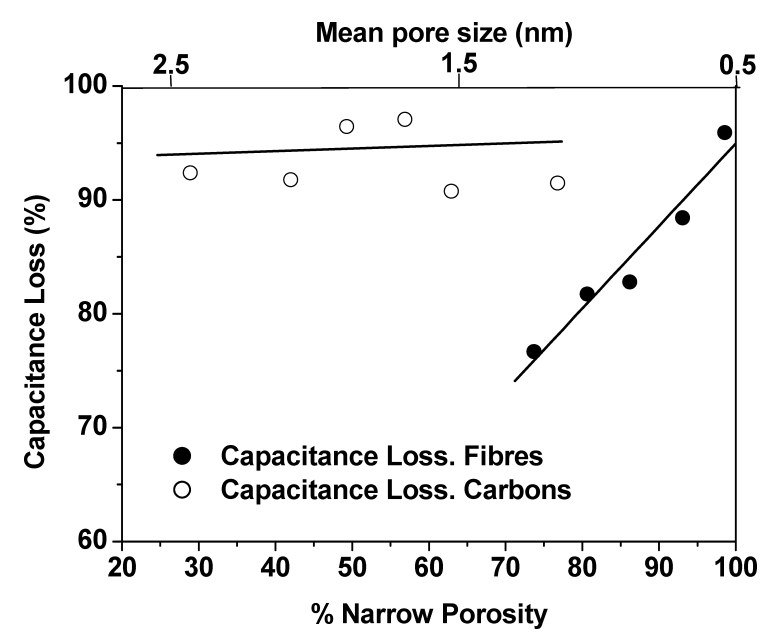

Fig. 7 Capacitance loss percentages (from the starting value to the value at around $0.02 \mathrm{~Hz}$ ) versus the percentage of narrow microporosity and average pore size for several ACs and ACFs in $1 \mathrm{M} \mathrm{Na}_{2} \mathrm{SO}_{4}$ solution.

haviour. However, in this electrolyte, the improvement is much more important than in $\mathrm{Na}_{2} \mathrm{SO}_{4}$ medium. These facts can be due to the higher mobility of proton in aqueous solution compared to any other ion because of its different charge-transfer mechanism.

Thus, from this systematic study it is concluded that kinetics of the double-layer formation in porous carbons is improved using: (i) samples with wide micropore size distribution, (ii) samples with a porous texture with low tortuosity (ACF), especially in $\mathrm{Na}_{2} \mathrm{SO}_{4}$ medium, and (iii) acidic medium as the electrolyte.

\section{Polyaniline/porous carbon electrodes by chemical and electrochemical polymerization}

Another way to improve the performance of porous carbon as supercapacitors is to prepare carbon based composites with materials that may add pseudocapacitance. An example for this possibility are carbon/conducting polymer composites, which can take the advantage from both, the double layer mechanism provided by porous carbon materials and the pseudo-capacitance contribution from conducting polymers. Our research group has prepared polyaniline (PANI)/ porous carbon composites by both chemical ${ }^{16)}$ and electrochemical polymerization methods ${ }^{17)}$ and those composites were characterized in terms of porosity and performance as electrochemical capacitors.

In the case of chemical polymerization, two methods were used to obtain the electrodes: (i) mixing, directly, the AC or ACF with chemically polymerized PANI; (ii) mixing the AC or ACF with aniline and subsequent chemical polymerization. In this second method, the surface chemistry of the AC plays a key role in the PANI coating. From the results of that experimental work it can be said that carbon/PANI composites are more effective as capacitors if the two components are mixed directly or polymerized over a carbon material with a low content in surface oxygen complexes. The second method using a 
thermally treated carbon (to remove surface oxygen groups) seems to be the best one. This method is very efficient to improve PANI contribution to the total capacitance of the material compared to the pure PANI. It is remarkable that small additions of PANI (about $6 \mathrm{wt} \%$ ) can produce an increase in capacitance of about $20 \%$ of the pristine $\mathrm{AC}$ (from 125 to $148 \mathrm{~F} / \mathrm{g}$ ).

Preliminary experiments carried out by position-resolved microbeam small angle X-ray scattering ( $\mu \mathrm{SAXS}$ ) on ACF/PANI composites reveal that for both preparation methods (chemical and electrochemical) the coating of PANI takes place inside the porosity and is higher in the external regions of the ACF than in the core. The fibre diameter is not considerably changed, and it seems that the penetration of PANI inside the fibres occurs in a larger extension for the chemical polymerization.

\section{Polyaniline/silica electrodes prepared by sol-gel methods and electrochemical polymerization}

In order to improve the performance of supercapacitors incorporating species providing pseudocapacitance, we have studied the possibility of tailoring the electroactive area of those elements by preparing hybrid materials. The pseudocapacitance of PANI can be tailored by its template growth through porous silica films prepared by sol-gel methodology ${ }^{18), 19)}$.

The PANI-silica composites are prepared by sequential deposition of silica followed by electrochemical reactive insertion of the aniline monomers through the silica pores. In that manner the dielectric silica acts as template for the growth of conductive PANI.

Different methods can be used for the deposition of silica on proper electrodes. It can be deposited by conventional methods using techniques as dip-coating or spin-coating from precursor sols, generally metastable acidic hydroalcoholic solutions of tetraethyl-orthosilicate $^{18)}$. However, an attractive alternative is the electroassisted deposition of silica from a precursor sol-gel solution that contains a supporting electrolyte. The application of a negative potential high enough to produce the solvent reduction produces the consumption of protons in the vicinity of the electrode. Such increase of the $\mathrm{pH}$ nearby the electrode accelerates the condensation of the silica colloids that remain deposited in the electrode. The modifications of the applied potentials and time of deposition allows to control and to tune the properties of the deposited silica ${ }^{19)}$.

The silica-modified electrodes can be then used as a porous matrix for the electrochemical growth of PANI. These electrodes are immersed in a solution containing aniline, which can diffuse within the silica pores to reach the conductive electrode surface. The growth of PANI can be performed by potentiodynamic or potentiostatic methods.

Fig. 8 shows a SEM image of a silica-PANI hybrid synthesized by potentiostatic deposition over a silica modified electrode. In this fig-

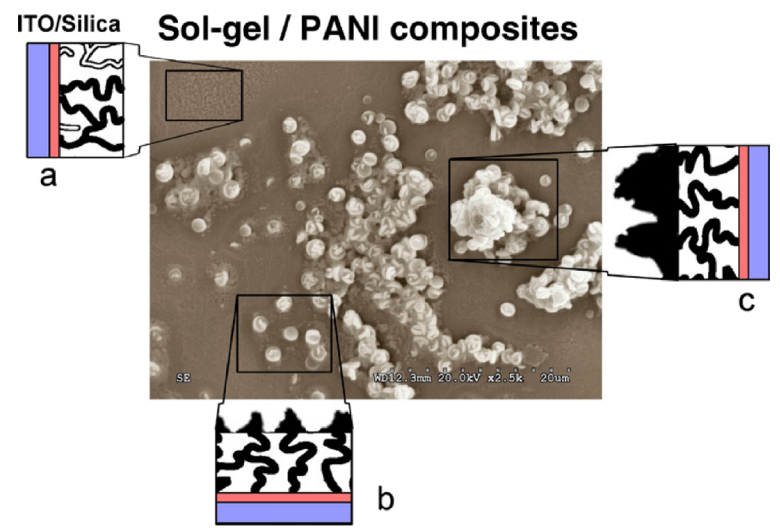

Fig 8 SEM image of a ITO/silica-modified electrode showing PANI emerging from the silica pores at different growing stages, as depicted in the schematic profiles of the electrode (marked as a, b, c).

ure schematic profiles (marked as a, b, c) of the electrodes show the presence of PANI fibres at different stages of growing through the silica pores. During the initial steps of electrochemical polymerization, PANI is confined inside the silica matrix (Fig. 8a) and the kinetics appears to be controlled by the hindered diffusion of aniline monomer though the silica pores. Later, once the PANI fibres have reached enough length, they emerge out of the template and the growth kinetics becomes comparable to that for PANI synthesized on a bare electrode (Fig. 8 b and c).

The silica matrix has a positive effect on the electrical capacitance of PANI since the polymer chains grow dispersed and the collapse between them is minimized. In that manner, the electrochemical capacitance of the Silica-PANI hybrids is several times higher than the capacitance of PANI synthesized at the same conditions. The silica matrix avoids the electric collapse between vicinal conducting fibres in ITO/Silica-PANI but allows the diffusion of ionic species that are in contact with the conjugated polymer, increasing therefore the conducting surface exposed to the electrolytic solution. The mass capacitance obtained for these hybrid materials is around $600 \mathrm{Fg}^{-1}$.

\section{Concluding Remarks}

A summary of the preparation of activated carbons (ACs) by chemical activation with alkaline hydroxides has been presented. It has been shown that, with careful control and thorough understanding of the variables affecting the carbon activation process, materials with different properties (apparent surface area, pore volume, pore size distribution, surface chemistry and porous structure) can be prepared. Examples discussing the importance of each of these properties on the double layer capacitance, on the kinetics of the electric doublelayer charge-discharge process and on the pseudo-capacitance contribution have been included. 


\section{Acknowledgments}

The authors would like to thank the Spanish Ministerio de Ciencia e Innovación, FEDER and PLAN E funds (MAT2010-15273 and PLE2009-0021) and Generalitat Valenciana (PROMETEO/2009/047) for financial support.

\section{References}

1) B. E. Conway, Electrochemical capacitors. (1999) New York: Kluwer Academic/Plenum Publishers.

2) A. Linares-Solano, D. Lozano-Castello, M. A. Lillo-Ródenas and D. Cazorla-Amorós, Chemistry and Physics of Carbon, Vol. 30 (L. R. Radovic, ed.), (2008) pp.1-62, Marcel Dekker: New York.

3) M. A. Lillo-Ródenas, J. P. Marco-Lozar, D. Cazorla-Amorós and A. Linares-Solano, J. Anal. Appl. Pyrolysis 80 (2007) 166-174.

4) D. Lozano-Castello, D. Cazorla-Amorós, A. Linares-Solano, S. Shiraishi, H. Kurihara and A. Oya, Carbon 41 (2003) 1765-1775.

5) S. Shiraishi, H. Kurihara and A. Oya, Electrochemistry 69 (2001) 440443.

6) S. Yoon, S. Lim, Y. Song, Y. Ota, W. Qiao, A. Tanaka and I. Mochida, Carbon 42 (2004) 1723-1729.

7) H. Teng, Y. Chang and C. Hsieh, Carbon 39 (2001) 1981-1987.
8) M. J. Bleda-Martínez, J. A. Maciá-Agulló, D. Lozano-Castelló, E. Morallón, D. Cazorla-Amorós and A. Linares-Solano, Carbon 43 (2005) 26772684.

9) H. Shi, Electrochim. Acta 41 (1996) 1633-1639.

10) E. Raymundo-Piñero, K. Kierzek, J. Machnikowski and F. Beguin, Carbon 44 (2006) 2498-2507.

11) M. C. Román, D. Cazorla-Amoros, A. Linares-Solano and C. SalinasMartínez de Lecea, Carbon 31 (1993) 895-902.

12) C. Hsieh and H. Teng, Carbon 40 (2002) 667-674.

13) K. Okajima, K. Ohta and M. Sudoh, Electrochim. Acta 50 (2005) 22272231.

14) M. J. Bleda-Martínez, D. Lozano-Castello, E. Morallón, D. CazorlaAmorós and A. Linares-Solano, Carbon 44 (2006) 2642-2651.

15) M. J. Bleda-Martínez, D. Lozano-Castello, D. Cazorla-Amorós and E. Morallón, Energy Fuels 24 (2010) 3378-3384.

16) M. J. Bleda-Martínez, E. Morallón and D. Cazorla-Amorós, Electrochim. Acta 52 (2007) 4962-4968.

17) M. J. Bleda-Martínez, C. Peng, S. Zhang, G. Z. Chen, E. Morallón and D. Cazorla-Amorós, J. Electrochem. Soc. 155 (2008) A672-A678.

18) F. Montilla, M. A. Cotarelo and E. Morallón, J. Mater. Chem. 19 (2009) 305-310.

19) D. Salinas-Torres, F. Huerta, F. Montilla and E. Morallón, Electrochim. Acta 56 (2011) 3620-3625. 